\title{
Withdrawal of benzodiazepines in elderly long term users does not produce significant adverse effects in the short term
}

Curran HV, Collins R, Fletcher S, et al. Older adults and withdrawal from benzodiazepine hypnotics in general practice: effects on cognitive function, sleep, mood and quality of life. Psychol Med 2003;33:1223-37.

\section{Does benzodiazepine withdrawal alter cognitive function, quality of life, mood, or sleep?}

\section{METHODS}

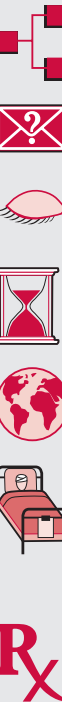

Design: Randomised controlled trial.

Allocation: Unclear.

Blinding: Double blind.

Follow up period: Twelve weeks.

Setting: Twenty five general practices in London and rural areas, UK; time period not specified.

Patients: 104 long term ( $>6$ months) users of benzodiazepines aged $\geqslant 65$ years (mean age 77 years) consenting to withdrawal. Exclusions: dementia or organic cognitive dysfunction; severe deafness or visual impairment; ongoing major psychiatric disorder; history of seizures; terminal illness.

R Intervention: Tapering doses of benzodiazepines for 8-9 weeks followed by placebo until week 24 ; usual benzodiazepine dose for 3 months, then tapering doses followed by placebo.

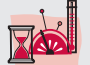

Outcomes: Cognitive and psychomotor function (range of tasks, including subtests from Wechsler Adult Intelligence Scale, Rivermead Behavioural Memory test, and Tests of Everyday Aftention); quality of life (SF-36); Benzodiazepine Withdrawal Symptom questionnaire (BWSQ) score; Geriatric Depression Scale (GDS) scores; mood rating scale scores; somatic symptom scores; sleep patterns (sleep diary and VAS scales).

$\stackrel{\square}{\longrightarrow \longrightarrow}$

Patient follow up: $87.5 \%$.

\section{MAIN RESULTS}

After six months, successful withdrawal from benzodiazepines was achieved by $80 \%$ of participants, with no significant difference between groups. There was no significant difference between groups in cognition, psychomotor function, mood, somatic symptoms, time spent in bed, number of awakenings per night, or difficulties in sleeping by sleep diary at 12 weeks ( 3 weeks after complete withdrawal in the discontinuation group; $\mathrm{p}$ values not given). Quality of life outcomes were similar between groups on SF-36 subscales.

For correspondence: Dr H V Curran, Psychopharmacology Research Unit, Clinical Health Psychology, University College London, London UK

Sources of funding: NHS Executive London (NHSE-LRO) Research and Development, Responsive Funding Programme.

\section{CONCLUSIONS}

In elderly long term users of benzodiazepines, withdrawal does not give rise to significant sleep disruption or withdrawal symptoms.

\section{NOTES}

This trial included a third treatment group, which did not agree to discontinue benzodiazepines. This group is not discussed here because it was not randomly selected.

\section{Commentary}

B enzodiazepines are the most widely used psychotropic drug worldwide. They do have some documented short term benefits: for adults with insomnia, a systematic review ${ }^{1}$ has found approximately 4 minutes reduction in sleep latency and 62 minutes increase in total sleep time on benzodiazepines compared with placebo. There were, however, only a small number of studies to ascertain this benefit among older subjects.

On the other hand, disadvantages of benzodiazepines are well established and numerous. They are clearly associated with certain adverse effects, including daytime drowsiness and motor and cognitive impairments. ${ }^{2}$ They can induce dependence in almost one third of patients taking them for four weeks or longer. Studies of putatively dependent patients suggest that, once dependence develops, only about half of those who try can successfully discontinue them. ${ }^{3}$ Benzodiazepines are also believed to be subject to development of tolerance, which is a decline in a drug's effects over time.

Benzodiazepines have also been associated with accident proneness. A cohort study focusing on hospitalisation caused by a fall revealed an NNH around 110-190 among the elderly. ${ }^{4}$ Another cohort study on motor vehicle accidents ${ }^{5}$ suggests that approximately 2900 people need to be treated with a benzodiazepine for two months in order to cause one hospitalisation due to traffic accidents ( $N N H=2860,95 \% \mathrm{Cl} 2050$ to 4760).

Is it then not a logical, pragmatic, and even ethical requirement that we conduct a randomised trial to see if elderly long term benzodiazepine users could not only be tapered off medication but also could show amelioration in motor and cognitive functioning without deterioration in sleep? Curran et al examined just this, and found that a great majority could be successfully withdrawn, with improvement in cognitive/motor tasks and without sleep or withdrawal problems. The study needs replication, though, because the dropout rates were rather high, rendering the interpretation of the obtained results less definitive.

Toshi A Furukawa, MD PhD Nagoya City University, Nagoya, Japan

1 Holbrook AM, Crowther R, Lotter A, et al. Meta-analysis of benzodiazepine use in the treatment of insomnia. CMAJ 2000;162:225-33.

2 Barker MJ, Greenwood KM, Jackson M, et al. Cognitive effects of long-term benzodiazepine use: a meta-analysis. CNS Drugs 2004;18:37-48.

3 Schweizer E, Rickels K. Benzodiazepine dependence and withdrawal: a review of the syndrome and its clinical management. Acta Psychiatr Scand Suppl 1998:393:95-101.

4 Neutel Cl, Hirdes JP, Maxwell CJ, et al. New evidence on benzodiazepine use and falls: the time factor. Age Ageing 1996;25:273-8.

5 Neutel Cl. Risk of traffic accident injury after a prescription for a benzodiazepine. Ann Epidemiol 1995:5:239-44. 